\title{
THE
}

1996

\section{Radiative Corrections and Quantum Chaos}

Yuri A. Dabaghian

University of Rhode Island

Follow this and additional works at: https://digitalcommons.uri.edu/phys_facpubs

Terms of Use

All rights reserved under copyright.

\section{Citation/Publisher Attribution}

Dabaghian, Y. A. (1996). Radiative Corrections and Quantum Chaos. Phys. Rev. Lett., 77(13), 2666-2669. doi: 10.1103/PhysRevLett.77.2666.

Available at: https://doi.org/10.1103/PhysRevLett.77.2666

This Article is brought to you for free and open access by the Physics at DigitalCommons@URI. It has been accepted for inclusion in Physics Faculty Publications by an authorized administrator of DigitalCommons@URI. For more information, please contact digitalcommons-group@uri.edu. 


\title{
Radiative Corrections and Quantum Chaos
}

\author{
Yuri A. Dabaghian \\ Department of Physics, University of Rhode Island, Kingston, Rhode Island 02881
}

(Received 4 June 1996)

\begin{abstract}
This Letter discusses the question of how the white noise of radiative corrections affects the chaotic properties of an original quantum system. It is shown, by an explicit mathematical analysis, that the radiative corrections, in effect, remove the original chaos in the system. [S0031-9007(96)01180-5]
\end{abstract}

PACS numbers: 05.45.+b, 03.65.Sq, 05.40.+j, 11.10.-Z

One of the most interesting questions in the theory of quantum chaotic systems is the question about the interrelations between the chaos and the quantum fluctuations. This question will be studied below in the example of a new model of quantum chaos, suggested recently in Ref. [1]. It will be demonstrated that the radiative corrections can suppress the chaos found in the exact QED Green's functions of potential theory. The possible mechanism of chaos suppression is discussed below in detail.

The chaotic behavior of the quantum system can be understood by analyzing the expression for the Green's function of the system:

$$
\begin{aligned}
G^{(0)}\left(x, y \mid A^{0}\right)= & \int_{0}^{\infty} d \tau e^{-i \tau m}[d X] F(X, \tau) \\
& \times \delta\left(\frac{d X_{\mu}}{d \tau}-\frac{1}{m} P_{\mu}+\frac{g}{m} A_{\mu}^{0}(X)\right),
\end{aligned}
$$

which is equivalent to what was obtained in [1] using the Fradkin representation. Here $\tau$ is the "proper time" of the particle, and the exact form of $F(X, \tau)$ is specified and discussed in Ref. [2]. The important feature of (1) is that this expression contains explicitly the $\delta\left(\frac{d X_{\mu}}{d \tau}-\frac{1}{m} P_{\mu}-\frac{g}{m} A_{\mu}^{0}(X)\right)$ under the integral, so that the functional integration over the trajectories $X_{\mu}(\tau)$ actually goes over the solutions to the effective dynamical system:

$$
m \frac{d X_{\mu}}{d \tau}=P_{\mu}(\tau)-g A_{\mu}^{0}(X) .
$$

Analyzing the behavior of that dynamical system, one can make some interesting statements about the quantum system described by the Green's function $G^{(0)}\left(A^{0}\right)$. For instance, if the system (2) is chaotic then (see [2]) the Green's function unavoidably inherits ultrasensitive dependence on the initial conditions, which are expressed in terms of functional variable $P_{\mu}(\tau)$. This situation, as shown in [1] and [2], can be naturally interpreted as chaos in a quantum system.

The dynamical system (1) describes the motion of a particle of "momentum" $P_{\mu}(\tau)$ in an electromagnetic field in terms of its vector potential. This is analogous to the relation between the generalized momentum of a particle and it's velocity, $m \frac{d X_{\mu}}{d \tau}+g A_{\mu}^{0}(X)=P_{\mu}$, for the particle that moves along the geodesics of the connection $A_{\mu}^{0}$. The interesting question is: If this is really a quantum chaos system, then how can the quantum fluctuations (the radiative corrections), which naturally appear in a real physical system, change the result?

The system with quenched radiative corrections is described by the Green's function $G$,

$$
G=\left.e^{-(i / 2) \int\left(\delta / \delta A_{\mu}\right) D_{\mu \nu}\left(\delta / \delta A_{\mu}\right)} G^{(0)}\right|_{A \rightarrow 0},
$$

which, as shown in [1], can be presented in the form

$$
\begin{aligned}
G= & \int[d X] d \tau e^{-i \tau m} e^{-\left(1 / g^{2}\right) \int_{0}^{\tau} \int_{0}^{\tau} d \tau_{1} d \tau_{2}\left[d X_{\mu} / d \tau_{1}-(1 / m) P_{\mu}-(g / m) A_{\mu}^{0}(X)\right]\left\langle\tau_{1}\left|K_{\mu \nu}^{-1}\right| \tau_{2}\right\rangle\left[d X_{\mu} / d \tau_{2}-(1 / m) P_{\mu}-(g / m) A_{\mu}^{0}(X)\right]} \\
& \times F(X, \tau) \frac{1}{g}\left(\operatorname{det} K_{\mu \nu}^{-1}\right)^{1 / 2},
\end{aligned}
$$

where $\left\langle\tau_{1}\left|K_{\mu \nu}\right| \tau_{2}\right\rangle=g^{2} D_{\mu \nu}\left(\tau_{1}, \tau_{2}\right)$, and $D_{\mu \nu}\left(\tau_{1}, \tau_{2}\right)$ is the photon propagator. So the $\delta$ functional is replaced by a smooth Gaussian distribution. Seemingly, the previous analysis is not applicable anymore to the system, as far as we are not restricted to the solutions of any dynamical system; and, consequently, one cannot ascribe dynamic Lyapunov exponents to the paths, as in (1). But, actually, using a simple transformation, one can restore the $\delta$ functional in the expression for the Green's function. Let us write the expression for $G$ in the form

$$
\begin{aligned}
G= & \int[d A] \iint d \tau e^{-i \tau m} e^{-\left(1 / g^{2}\right) \int_{0}^{\tau} \int_{0}^{\tau} d \tau_{1} d \tau_{2}\left(A_{\mu}-A_{\mu}^{0}\right)\left\langle\tau_{1}\left|K_{\mu \nu}^{-1}\right| \tau_{2}\right\rangle\left(A_{\nu}-A_{\nu}^{0}\right)} \\
& \times\left(\operatorname{det} K_{\mu \nu}^{-1}\right)^{1 / 2} \delta\left(\frac{d X_{\mu}}{d \tau}-\frac{1}{m} P_{\mu}-\frac{g}{m} A_{\mu}(X)\right) F(X, \tau)[d X] .
\end{aligned}
$$


Here we have introduced a new functional variable $A_{\mu}$, and an additional integration over it, which due to the $\delta\left(\frac{d X_{\mu}}{d \tau}-\frac{1}{m} P_{\mu}-\frac{g}{m} A_{\mu}(X)\right)$ is trivial, can be performed explicitly, and evidently does not change the result. But now the last three terms $\tilde{G}^{(0)}\left(x, y, \tau \mid A^{0}\right) \equiv \int \delta\left[\frac{d X_{\mu}}{d \tau}-\right.$ $\left.\frac{1}{m} P_{\mu}-\frac{g}{m} A_{\mu}(X)\right] F(X, \tau)[d X]$ in (3) resemble the initial expression for the Green's function, $G^{(0)}(A)$, without the radiative corrections, although defined on the solutions to some other dynamical system. It should be mentioned that, as far as we have only one integration over the "time" $\tau$ for the whole ensemble, the $\tilde{G}^{(0)}$ 's are not exactly the Green's functions of the systems. In other words, the systems in the ensemble are not independent, the Gaussian distribution mixes them up. The variable $A$ introduced in (3) would stand for the "force function" of that dynamical system,

$$
\frac{d X_{\mu}}{d \tau}=\frac{1}{m} P_{\mu}-\frac{g}{m} A_{\mu}(X) .
$$

The Green's function then becomes

$$
\begin{aligned}
G(x, y)= & \int e^{-\left(1 / g^{2}\right) \int_{0}^{\tau} \int_{0}^{\tau} d \tau_{1} d \tau_{2}\left(A_{\mu}-A_{\mu}^{0}\right)\left\langle\tau_{1}\left|K_{\mu \nu}\right| \tau_{2}\right\rangle\left(A_{\nu}-A_{\nu}^{0}\right)} \\
& \times\left(\operatorname{det} K_{\mu \nu}\right)^{1 / 2} \tilde{G}^{(0)}(x, y, \tau \mid A)[d A]
\end{aligned}
$$

One can see from this expression that the total $G$ is a result of the averaging over the infinite ensemble of dynamical systems, where each system has its own force function $A_{\mu}$, and that these $A_{\mu}$ 's are Gaussian distributed around the initial system (1) described by $A_{\mu}^{0}$. To understand the dynamical properties of the ensemble, we have to understand how "chaotic" a typical system in the ensemble is, and how the different systems contribute to the whole picture. Although the force functions $A_{\mu}$ are " $g$ close" to the original chaotic $A_{\mu}^{0}$ in the sense of the distribution (5), some of the systems in the ensemble are certainly integrable; even more, it is well known that integrable trajectories are dense around each nonintegrable trajectory, so these functions $A_{\mu}$, which correspond to the integrable systems, are not rare [3].

According to the general principles of quantum mechanics, in order to find the probability of a particle to go from point $x$ to point $y$, one must add the probability amplitudes of the transition, and calculate the square of the modulus of this sum:

$$
\begin{aligned}
w & \sim\left|\sum_{i} \psi_{i}(x \rightarrow y)\right|^{2} \\
& =\sum_{i}\left|\psi_{i}(x \rightarrow y)\right|^{2}+\sum_{i \neq j} \psi_{i}(x \rightarrow y) \psi_{j}^{*}(x \rightarrow y) .
\end{aligned}
$$

The first term on the right hand side gives the probability flow along each individual amplitude, and the second one is the interference term. In our case, if one takes the square of modulus of the expression (5), and retains only the term that describes the probability flow along each system, then

$$
\begin{aligned}
w= & \int e^{-\left(1 / g^{2}\right) \int_{0}^{\tau_{1}} \int_{0}^{\tau_{1}} d \tau_{1}^{\prime} d \tau_{2}^{\prime}\left\langle\tau_{1}^{\prime}\left|K_{\mu \nu}\right| \tau_{2}^{\prime}\right\rangle\left(A_{\nu}-A_{\nu}^{0}\right)_{1}+\left(1 / g^{2}\right) \int_{0}^{\tau_{2}} \int_{0}^{\tau_{2}} d \tau_{1}^{\prime} d \tau_{2}^{\prime}\left(A_{\mu}-A_{\mu}^{0}\right)_{2}\left\langle\tau_{1}^{\prime}\left|K_{\mu \nu}\right| \tau_{2}^{\prime}\right\rangle\left(A_{\nu}-A_{\nu}^{0}\right)_{2}} \\
& \times\left.\left(\operatorname{det} K_{\mu \nu}\right) \tilde{G}\left(x, y, \tau_{1} \mid A_{1}\right) \tilde{G}^{*}\left(x, y, \tau_{2} \mid A_{2}\right)\left[d A_{1}\right]\left[d A_{2}\right]\right|_{A_{1}=A_{2}} \\
= & \int e^{-\left(1 / g^{2}\right) \int_{\tau_{2}}^{\tau_{1}} \int_{\tau_{2}}^{\tau_{1}} d \tau_{1}^{\prime} d \tau_{2}^{\prime}\left(A_{\mu}-A_{\mu}^{0}\right)\left\langle\tau_{1}^{\prime}\left|K_{\mu \nu}\right| \tau_{2}^{\prime}\right\rangle\left(A_{\nu}-A_{\nu}^{0}\right)}\left(\operatorname{det} K_{\mu \nu}\right)^{1 / 2} \tilde{G}\left(x, y, \tau_{1} \mid A\right) \tilde{G}^{*}\left(x, y, \tau_{2} \mid A\right)[d A] .
\end{aligned}
$$

The variables $\left[d A_{1}\right]$ and $\left[d A_{2}\right]$, which appear in the second order functional integral for $w$, are defined over the different intervals of time, so for the diagonal part of the sum, when $A_{1}=A_{2}$, the probability $w$ can be represented as a single integration over the variable $[d A]$, which is the same over the whole time scale. The evaluation of the determinant $\operatorname{det} K_{\mu \nu}$ in (6) is different from that in (5) (see [4]), so it retains its power 1/2.

The interference is not important for most of the amplitudes, since the lengths of the trajectories for different systems and hence their phases are very different, so the main contribution to the probability sum should come from (6), and this is the only contribution considered here. In the Fradkin representation [5] the explicit expression for the Green's function $G^{(0)}(x, y \mid A)$ has the form

$$
\begin{aligned}
G^{(0)}(x, y \mid A)= & i \int_{0}^{\infty} d \tau \int[d v] \\
& \times e^{-i \tau m} e^{i \int_{0}^{\tau} d \tau^{\prime} v_{\mu}^{2}\left(\tau^{\prime}\right)}\left[m-\gamma_{\mu} v_{\mu}(\tau)\right] e^{-i g \int_{0}^{\tau} d \tau^{\prime} v_{\mu}\left(\tau^{\prime}\right) A_{\mu}\left(y-\int_{0}^{\tau^{\prime}} v\right)} \\
& \times\left(e^{g \int_{0}^{\tau} d \tau^{\prime} \sigma^{\mu \nu}\left(\tau^{\prime}\right) F_{\mu \nu}\left(y-\int_{0}^{\tau^{\prime}} v\right)}\right)_{+} \delta\left(x-y+\int_{0}^{\tau} v\right)
\end{aligned}
$$


Now, using the substitution $v_{\mu}=\frac{d X_{\mu}}{d \tau}$, and the "identity" $x-y+\int_{0}^{\tau} v d \tau^{\prime}=0$ (the argument of the $\delta$ function), one has

$$
\begin{aligned}
G^{(0)}(x, y \mid A)= & i \int_{0}^{\infty} d \tau e^{-i \tau m} e^{i \int_{y}^{x} v_{\mu} d X_{\mu}}\left[m-\gamma_{\mu} v_{\mu}(\tau)\right] \\
& \times e^{i \int_{y}^{x} A_{\mu} d X_{\mu}}\left(e^{g \int_{y}^{x} F_{\mu \nu}(X) \sigma^{\mu \nu} d X_{\mu}}\right)_{+}\left[d \gamma_{\tau}\right] .
\end{aligned}
$$

exponential terms of the previous expression we will have

$$
\begin{aligned}
G^{(0)}(x, y \mid A)= & i \int_{0}^{\infty} d \tau e^{-i \tau m} \int\left[m-\gamma_{\mu} v_{\mu}\right] \\
& \times e^{i \int_{y}^{x}\left[\left(d X_{\mu} / d \tau\right)+g A_{\mu}(X)\right] d X_{\mu}} \\
& \times\left(e^{g \int_{y}^{x} F_{\mu \nu}(X) \sigma^{\mu \nu} d X_{\mu}}\right)_{+}\left[d \gamma_{\tau}\right] .
\end{aligned}
$$

The integration in the exponents goes over a path $\gamma\left(\tau^{\prime}\right)$ such that $0 \leq \tau^{\prime} \leq \tau$, and $\left[d \gamma_{\tau}\right]$ represents the functional integration over all paths. Combining the first two
The function $\tilde{G}(x, y, \tau \mid A)$ has no integration over $\tau$, so we finally have for the $|\tilde{G}(x, y, \tau \mid A)|^{2}$ the following:

$$
\begin{aligned}
\tilde{G}\left(x, y, \tau_{1} \mid A\right) \tilde{G}^{*}\left(x, y, \tau_{2} \mid A\right)= & \iint\left[d \gamma_{1}\right]\left[d \gamma_{2}\right] e^{i\left(\tau_{1}-\tau_{2}\right) m}\left|m-\gamma_{\mu} v_{\mu}\right|^{2} \\
& \times e^{-i \int_{y}^{x\left(\tau_{1}\right)}\left[d X_{\mu} / d \tau+g A_{\mu}(X)\right]_{1} d X_{\mu}+i \int_{y}^{x\left(\tau_{2}\right)}\left[d X_{\mu} / d \tau+g A_{\mu}(X)\right]_{2} d X_{\mu}}\left|\left(e^{g \int_{y}^{x} F_{\mu \nu}(X) \sigma^{\mu \nu} d X_{\mu}}\right)_{+}\right|^{2} .
\end{aligned}
$$

The integration in the two different brackets in the exponent goes over two different paths $\gamma_{1}\left(\tau_{1}\right)$ and $\gamma_{2}\left(\tau_{2}\right)$.

In order to understand where the main contribution to the total probability comes from, we shall look at the saddle point of the expression (6). In Feynmann's formalism, a typical Green's function is obtained from the functional integral

$$
G(x, y)=\int e^{i S(x, y)} d[\gamma],
$$

where the "saddle point" of the functional $S$ is given by the classical solution $\frac{\delta S}{\delta x(t)}=0$. In the same way, in the expression $e^{-i \int_{y}^{x}\left[d X_{\mu} / d \tau+g A_{\mu}(X)\right] d X_{\mu}}=e^{-i \int_{y}^{x} P_{\mu} d X_{\mu}}$ the path that corresponds to the solution to $\frac{d X_{\mu}}{d \tau}+g A_{\mu}(X)=$ $P_{\mu}$ will extremize the functional. This means, in turn, that the exponent in the expression (9) finds it's extremum value on the two paths. If we change the order of integration in the second integral

$$
\begin{aligned}
i \int_{x(0)}^{y\left(\tau_{2}\right)}\left(\frac{d X_{\mu}}{d \tau}\right. & \left.+g A_{\mu}(X)\right) d X_{\mu} \\
& \longrightarrow-i \int_{y\left(\tau_{2}\right)}^{x(0)}\left(\frac{d X_{\mu}}{d \tau}+g A_{\mu}(X)\right) d X_{\mu},
\end{aligned}
$$

then the total argument of the exponents will be

$$
\begin{aligned}
\int_{x(0)}^{y\left(\tau_{1}\right)}\left(\frac{d X_{\mu}}{d \tau}\right. & \left.+g A_{\mu}(X)\right) d X_{\mu} \\
& +\int_{y\left(\tau_{2}\right)}^{x(0)}\left(\frac{d X_{\mu}}{d \tau}+g A_{\mu}(X)\right) d X_{\mu} .
\end{aligned}
$$

Here one can see the type of solutions needed to extremize the functional (9). One solution should start at the point $x$ and come to the point $y$ by the time $\tau_{1}$, and the other should go back from $y$ to $x$, starting at the time $\tau_{2}$. The "back path" $\gamma_{2}$ is different from $\gamma_{1}$. We did not change the sign of $\tau$, so $\gamma_{2}$ is not, generally speaking, $\gamma_{1}(-\tau)$. The system needs, indeed, two kinds of solutions to minimize (9). The possibility exists, for example, if the system (4) is integrable that it has closed periodic trajectories. In this case, the system can return back to the starting point, and then repeat this process infinitely many times. It's solutions simply wind around tori in the phase space and yield closed loops. Hence the integrable system can appear at the point $y$ (or $x$ ) at different moments of time $\tau_{2}, \tau_{3}, \ldots, \tau_{n}, \ldots$, and these moments of time are not arbitrary, since the system performs periodic motion. The characteristic frequencies of the system should satisfy

$$
\omega_{1} n_{1}+\omega_{2} n_{2}+\cdots+\omega_{k} n_{k}=0,
$$

which relation guarantees that there are closed-loop paths in the phase space. In the case when $\tau_{1}=\tau_{2}$ the sum of two exponents (10) gives a single integration over a closed loop. And when $\tau_{1}$ and $\tau_{2}$ are not equal, then between these moments of time the trajectory will simply run over the tori several times. Thus, for the integrable trajectories the sum of two exponents in (10) can be presented as an integration over a closed loop,

$$
\int_{x}^{y\left(\tau_{1}\right)}\left(\frac{d X_{\mu}}{d \tau}+g A_{\mu}\right) d X_{\mu}+\int_{y\left(\tau_{2} \neq \tau_{1}\right)}^{x}\left(\frac{d X_{\mu}}{d \tau}+g A_{\mu}\right) d X_{\mu}=n \oint_{C(T)}\left(\frac{d X_{\mu}}{d \tau}+g A_{\mu}\right) d X_{\mu},
$$


where $\oint_{C(T)}$ is the integration over the period, $A_{\mu}=$ $A_{\mu}\left(X_{\mu}\right)$. The number of times the solution winds around a torus, $n$, is just one of the quantum numbers of the system.

In any case, if we are looking for the saddle point trajectories for the expression (10), one must consider the solutions according to which system (4) appears twice at the same point, at the moments of time $\tau_{1}$ and $\tau_{2}$. This is actually a rather strong requirement that is imposed on the system. For instance, if a system is not completely integrable, then it has solutions which do not wind around any tori, they simply cover the whole phase space. If that kind of trajectory will appear in the vicinity of the same point more than once, it will do it, firstly, fairly irregularly, and, secondly, after a very long period of time, which is certainly longer than any characteristic time $\frac{1}{\omega}$ for most of the integrable trajectories [4]. For these reasons the trajectories that are chaotic will not give a serious contribution to the expression (9). The main contribution of (9) comes from the integrable trajectories of the system (1). In other words, the larger the amount of integrable trajectories of a system, the more will be their contribution in the sum (9) .

So, at this point, we can see that for each system in the expression (9) the majority of the trajectories will not give an important contribution. According to (9), the main contribution to the probability of a system which is not completely integrable (where some of it's trajectories are integrable and some are not) should flow along the integrable paths. The amount of integrable trajectories in the original chaotic system (2) is probably not that large, but the fluctuations of $A^{0}$ in phase space language produce something like "fluctuating tori," which appear and disappear all the time. These tori create additional integrable trajectories, and the biggest contribution in (9) comes exactly from these integrable, "fluctuating" trajectories in the ensemble. Thus the probability flows along the web of the "virtual," resonant tori in phase space, the tori for which the condition $\omega_{1} n_{1}+\omega_{2} n_{2}+\cdots+\omega_{k} n_{k}=$ 0 holds. Even the majority of the irrational tori drop off. An irrational torus should be "almost rational" in order to give a significant contribution. That is, if the "irrationality" of the torus can be approximated by a rational number, then the trajectories can appear in a given small vicinity of certain points rather regularly. On the other hand, one can say that a dynamical system is as much integrable as it has independent "closed loop" solutions. In this sense, the largest contribution to the integral (9) comes from the integrable systems of the ensemble.

There is a rather beautiful effect in classical mechanics known as "Arnold diffusion" [6]. A particle whose motion is not completely restricted by the integrals of motion "diffuses" in phase space in between the web of the rational tori. Formula (9) predicts, in fact, the same thing. A system will use the available tori in phase space to minimize the integral (9), but if the number of integrals is less than the number of degrees of freedom, part of it's trajectories will wander all over the phase space and yield some "diffusion." But once the radiative corrections are included, the situation becomes very different. According to the distribution (1), the particle could choose among the different trajectories - solutions to the system (2)but according to the distribution (6) it can now choose the system which provides the most suitable trajectories. Thus the particle tends to go along the "virtual tori," which appear as the result of quantum fluctuations in phase space. In the limit $g \rightarrow 0$, when the Gaussian distribution shrinks and yields the $\delta$ function of the original $A_{\mu}^{0}$ system, all field fluctuations disappear and so do the virtual tori; the particle starts to diffuse according to the Arnold scenario, and the system again becomes chaotic. That means that the "white noise of the radiative corrections," in effect, restructures the phase space, and, as a result, removes the original chaos of the system. Since these arguments lead to the conclusion that the magnitude $\left|G_{c}(x, y)\right|$ [of the two-point function containing quantum fluctuations of the field $A_{\mu}(z)$ appearing in the Green's function $G_{c}(x, y \mid A)$ ] should not be sensitive to chaotic fluctuations, it follows that chaos can appear only in the phase of this two-point function.

I am grateful to Professor H. M. Fried for introducing me to the problem and for stimulating discussions. I have also benefited greatly from the helpful suggestions and discussions of Professor A. E. Meyerovich.

[1] H. M Fried, Y. Gabelini, and B. H. J. McKellar, Phys. Rev. Lett. 74, 4373 (1995); H. M. Fried and Y. Gabelini, Phys. Rev. D 51, 890 (1995).

[2] H.M. Fried, Y. Gabelini, and B.H.J. McKellar, Phys. Rev. D 51, 7083 (1995).

[3] L.E. Reichl, The Transition to Chaos (Springer-Verlag, New York, 1992).

[4] M. C. Gutzwiller, Chaos in Classical and Quantum Mechanics (Springer-Verlag, Berlin, 1990).

[5] E.S. Fradkin, Nucl. Phys. 76, 588 (1966); H. M. Fried, Functional Methods and Eikonal Models (Edition Frontieres, Gif-sur-Yvette, France, 1990).

[6] V.I. Arnol'd, Russ. Math. Survey 18, 9 (1963); 18, 85 (1963); B. V. Chirikov, Phys. Rep. 52, 265 (1979). 